\title{
Human four-dimensional spatial intuition in virtual reality
}

\author{
Michael S. Ambinder, Ranyiao Frances Wang, \\ James A. Crowell, ANd GeOrge K. Francis \\ University of Illinois, Urbana-Champaign, Illinois
}

AND

Peter Brinkmann

City College, New York, New York

\begin{abstract}
It is a long-lasting question whether human beings, who evolved in a physical world of three dimensions, are capable of overcoming this fundamental limitation to develop an intuitive understanding of four-dimensional space. Techniques of analogy and graphical illustration have been developed with some subjective reports of success. However, there has been no objective evaluation of such achievements. Here, we show evidence that people with basic geometric knowledge can learn to make spatial judgments on the length of, and angle between, line segments embedded in four-dimensional space viewed in virtual reality with minimal exposure to the task and no feedback to their responses. Their judgments incorporated information from both the three-dimensional (3-D) projection and the fourth dimension, and the underlying representations were not algebraic in nature but based on visual imagery, although primitive and short lived. These results suggest that human spatial representations are not completely constrained by our evolution and development in a 3-D world. Illustration of the stimuli and experimental procedure (as video clips) and the instruction to participants (as a PDF file) may be downloaded from http://pbr.psychonomic-journals.org/content/supplemental.
\end{abstract}

Representations of space and time are deeply rooted in human thinking, reasoning, and perception of the world (Abbott, 1991; Bork, 1964; Durrell, 1938; Gardner, 1975; Kant, 1881/1896; Reichenbach, 1958; Rucker, 1984). However, living in a physical world of three dimensions, humans have their perceptual and cognitive systems tailored for sensing, storing, transforming, and reasoning about three-dimensional (3-D) objects. Some thinkers (e.g., Kant, 1881/1896) believe that space is an innate concept, whereas perceptual experience simply fills it with objects. Thus, humans may develop symbolic systems to conceptualize multidimensional entities that they call highdimensional space, but they can never overcome the innate constraint and add another dimension to the 3-D mental space that corresponds to the physical world, because they can never figure out where that dimension could be put.

Much effort has been made to challenge this cognitive limitation and to develop human four-dimensional (4-D) intuitions (Davis, Hersh, \& Marchisotto, 1995; Gardner, 1969; Rucker, 1984; Seyranian, 2001; Weeks, 1985). Two basic techniques were proposed to help people obtain an intuition of 4-D space. The first is by analogy to 3-D space. This technique has been widely used. For example, Berger
(1965; Abbott, 1991) explained how a 4-D creature can enter a 3-D locked closet from the fourth dimension by describing how a 3-D creature enters a two-dimensional (2-D) enclosure from above without touching its walls.

The second technique is to lift an observer into the higher dimensional space, so that he or she can directly experience it perceptually (Abbott, 1991; Berger, 1965; Rucker, 1984; Seyranian, 2001). For example, Abbott suggested that a 2-D creature can obtain 3-D intuition when it is taken into the 3-D space and views its world from above. Although this approach is hypothesized to be the most powerful means of acquiring 4-D intuition, it was not possible to implement the technique until virtual reality was available (D'Zmura, Colantoni, \& Seyranian, 2000; Francis, 2005).

There have been informal subjective reports that mathematicians who actively interacted with computergraphical simulations of 4-D geometric objects obtained sudden, novel insights of higher dimensional space (e.g., Davis et al., 1995). However, there has been no objective evaluation of such achievements. In the present study, we examined the dimensionality limitation in human spatial representations by constructing 4-D geometric objects in virtual reality and measuring judgments of distance

R. F. Wang, francesw@cyrus.psych.uiuc.edu 
(a property of one-dimensional [1-D] space) and angle (a property of 2-D space), which are spatial properties of the lowest dimensions and serve as the building blocks of higher dimensional spatial properties. If the participants can learn 4-D spatial relationships, their judgments should correlate with the actual distances and angles.

\section{METHOD}

\section{Participants}

Four participants were recruited from the Department of Psychology at the University of Illinois at Urbana-Champaign. Half of them were female. Three were graduate students and naive to the design of the study.

\section{Apparatus}

The experiment was conducted in the six-sided, fully immersive Beckman Institute Virtual Reality Cube ${ }^{1}$ at the University of Illinois at Urbana-Champaign. Each surface of the Cube is a rear-projection screen. Stereovision was achieved through a Stereographics LCD shutter glass system synchronized with the projector and computer graphics. A wireless Ascension MotionStar tracking system determined the six degrees of freedom positions and the orientation of the participant's head and of a gamepad in order to render the appropriate images.

\section{Stimuli}

The stimuli were randomly shaped hypertetrahedrons (four simplexes). Each hypertetrahedron was constructed with five 4-D points as the vertices. All coordinates were randomly chosen between $-0.6 \mathrm{~m}$ and $0.6 \mathrm{~m}$ with certain constraints (see the Component Analysis section below). Then all points were connected to all others, and all triangular faces were filled.

The 4-D object was displayed using a slicing technique (Seyranian, 2001). Figures $1 \mathrm{~A}$ and $1 \mathrm{~B}$ show an analogy of the technique in three dimensions. In principle, a 2-D creature can build a mental representation of the 3-D object by integrating the 2-D slices that it sees over time, although it does not know where that third dimension is in physical space (Abbott, 1991). Similarly, our participants studied the 4-D objects by walking along the $w$-axis, which revealed its 3-D sections successively. The 3-D cross-sections of these hypertetrahedrons were wire-frame objects that might take the shape of a single point, a tetrahedron, or a triangular prism (see Figure 2A).

For each object, three of the five vertices were randomly selected as the target vertices. Whenever a target vertex entered the 3-D observation chamber, a small cube (a marker) with a corresponding texture on its faces appeared at the location of its entrance. The marker disappeared when the vertex left the observation chamber.

\section{Procedure}

Each participant was given a brief lecture before the experiment on the structure of both 4-D space and 4-D objects. Analogies were drawn to studying 3 -D objects through a 2 -D viewing window. A summary of the lecture can be found online at http://pbr.psychonomic -journals.org/content/supplemental. After we had ensured that the participants understood the nature of 4-D space, the participants were tested in two tasks, in one session for each task. In each session, they had a 15 -min practice followed by approximately 20 experimental trials.

In each trial, the participants held a gamepad with a tracker attached and were instructed to walk forward and backward along the $z$-axis toward an object in order to change their position in the $w$-direction (Figure 2B). The simulated position of the observer along the $w$-axis was determined by the $z$ coordinates of the gamepad. That is, a 1-m displacement of the gamepad in the $z$ direction led to a 1-m displacement of the observation chamber in the $w$ direction. On average, the $3-\mathrm{D}$ slices were located $1.5 \mathrm{~m}$ from the observer. ${ }^{2}$ The participants studied the 3-D slices of a target object and were asked to mentally construct the 4-D object.

After a free viewing study period of a maximum of $2 \mathrm{~min}$, the object disappeared and the response stimuli appeared. In the distancejudgment task, a horizontal bar appeared with two patterned boxes on each end. The participant adjusted the length of the line using the gamepad to match the Euclidean distance between the two corresponding targets in 4-D space (Figure 2C). In the relativeorientation-judgment task, a "V" dial appeared with three markers. The participants judged the markers' relative orientation by adjusting the top line segments to match the angular relationship between the two arms in 4-D space (Figure 2D). The initial length/angle was determined by the orientation of the gamepad at the moment of the appearance of the stimulus and was thus random across trials. The actual distance in the distance-judgment task varied from 0.3 to $1.6 \mathrm{~m}$. The actual angles in 4-D space were between $10^{\circ}$ and $150^{\circ}$.

The only training that the participants received was a 15 -min practice on each task with the same type of stimuli to familiarize themselves with the apparatus and the procedure. No feedback of any kind was given in any of the trials. Thus, unlike those in previous research (Seyranian, 2001), the participants could not simply learn the relationship between the pattern of stimuli and the correct responses through trial and error. Instead, they had to grasp the 4-D structure
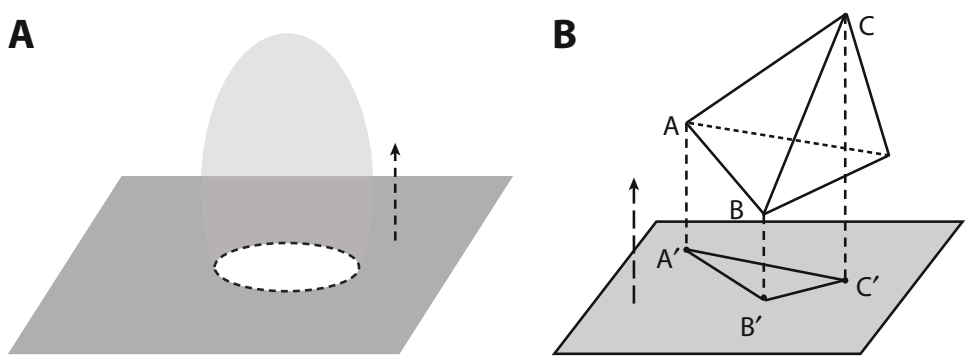

Figure 1. A three-dimensional (3-D) analogy of the slicing technique and the distance and relative-orientation judgments used in this study. (A) A twodimensional (2-D) observation space (dark gray plane) slicing through a 3-D object (light gray egg) along a direction orthogonal to the plane. Different horizontal slices of the egg pass through the observation space successively as it slides along the vertical direction. (B) A 3-D analogy of the component analysis. When a 2-D observation window (the gray plane) slides through a tetrahedron with three marked target vertices (e.g., A, B, and C), they enter the observation window successively. The points at which they each appear in the observation window form a triangle $\left(A^{\prime} B^{\prime} C^{\prime}\right)$. These points define the corresponding 2-D components of the length and angles in three dimensions. 
A
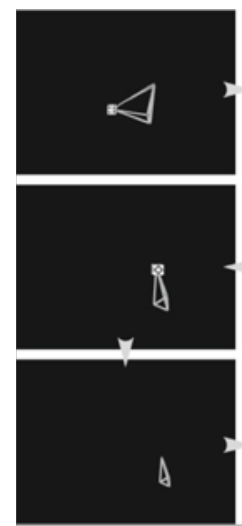

$\Delta$
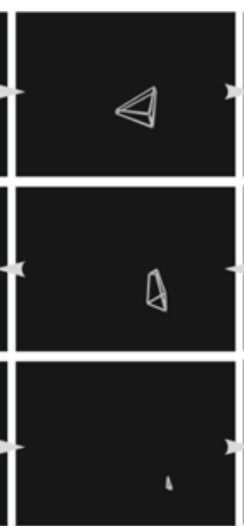

C

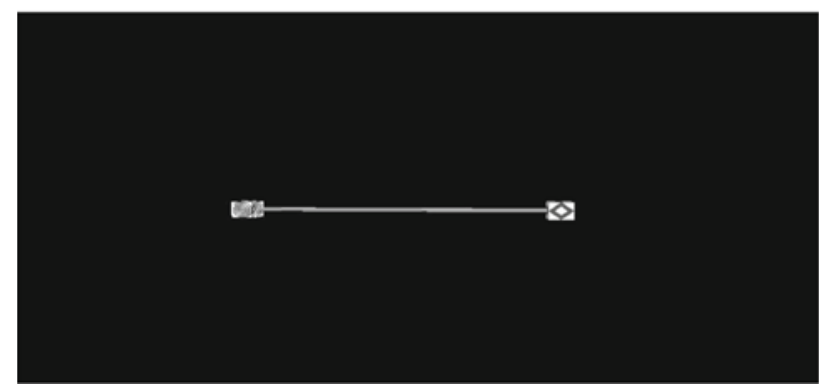

B
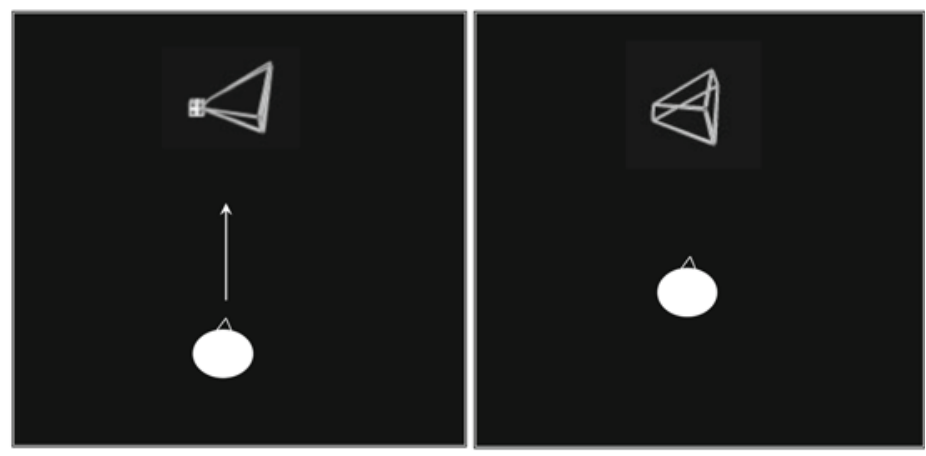

D

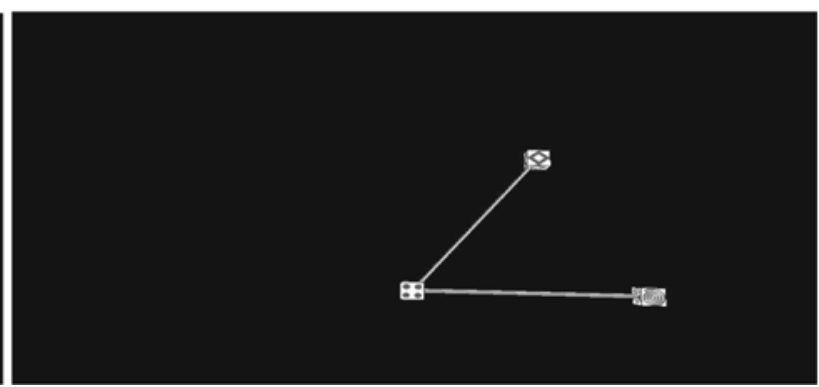

Figure 2. Sample test and response stimuli. An illustration of the stimuli and procedure in video format may be downloaded from http://pbr.psychonomic-journals.org/content/supplemental. (A)A sample sequence that the participants saw. (B) An overhead view of a participant studying a 4-D object by walking back and forth toward the object, which moves the observation chamber along the $w$-axis by the same distance. (C) The response stimuli for the distance-judgment task. (D) The response stimuli for the relative-orientationjudgment task.

from their visual experience itself. Moreover, the exact task in a particular trial (i.e., which of the three possible distances/angles was tested) was not given until the response period, further emphasizing the need for a spatial representation of the object rather than a direct estimation from perceptual cues during the learning period.

\section{Component Analysis}

As is illustrated in Figure 1B, a 2-D observer inside the gray plane may easily perceive triangle $\mathrm{A}^{\prime} \mathrm{B}^{\prime} \mathrm{C}^{\prime}$ over time. However, in order to determine the appropriate spatial relationships among $\mathrm{A}, \mathrm{B}$, and $\mathrm{C}$, it is necessary to make use of the additional information provided by the movement of the plane through 3-D space. Similarly, the participants in our study may also have easily perceived the location in which each vertex of a 4-D object appeared in their 3-D observation chamber. However, in order to judge the spatial relationship in 4-D, they had to use the additional information of their movements along the $w$-axis. These two types of information are orthogonal and can be measured independently.

We made use of this property to tease apart the sources of information that the participants used in making their judgments in our experiment. In three dimensions, the length of the segments and angles of triangle $\mathrm{A}^{\prime} \mathrm{B}^{\prime} \mathrm{C}^{\prime}$ are the 2-D components (Figure $1 \mathrm{~B}$ ), whereas the difference between the actual lengths/angles and their 2-D components, which is caused by their extension into the third dimension, can be defined as their 3-D component. Similarly, in four dimensions, the distances and angles can be decomposed into 3-D and 4-D components. The 3-D components represent the properties that one can obtain from the targets' entrance points inside the observation chamber, whereas the 4-D components represent the contribution of the 4-D structure. ${ }^{3}$ A multiple regression analysis of the responses as a function of the 3-D and 4-D components reveals the sources of information that the participants used for their judgments. ${ }^{4}$

\section{RESULTS}

The subjective reports obtained during a postexperiment interview suggest that the participants solved the task by first forming an image of the object in 3-D space (i.e., its 3-D projection) and then attempting to imagine an additional reference frame on which the 3-D representation of the object could expand or contract by the corresponding "hyperdepth" to obtain the real shape of the object. Thus, subjectively, all of the participants sought to use an imagery strategy (Finke, 1993; Kosslyn, 1994; Shepard \& Cooper, 1982) and to form visual-spatial representations in four dimensions.

More important, objective measurements of their performance suggest that this strategy was successful. In the distance-judgment task, all of the participants showed significant correlation between their responses and the actual 4 -D distance $(r \mathrm{~s}=.80, .66, .54, .90 ; t \mathrm{~s}>2.72 ; p \mathrm{~s}<.02$; see Figure 3). A multiple linear regression analysis using both 3-D and 4-D components as predictors of the actual response showed that the 4-D component was a significant predictor for all 4 participants $(\beta \mathrm{s}=.70, .55, .56$, 
F.W.

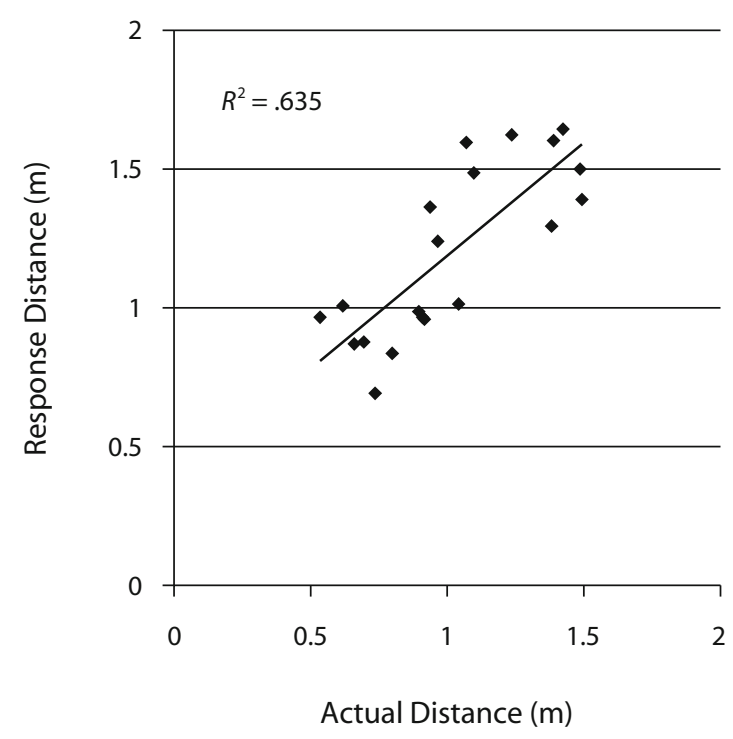

E.C.

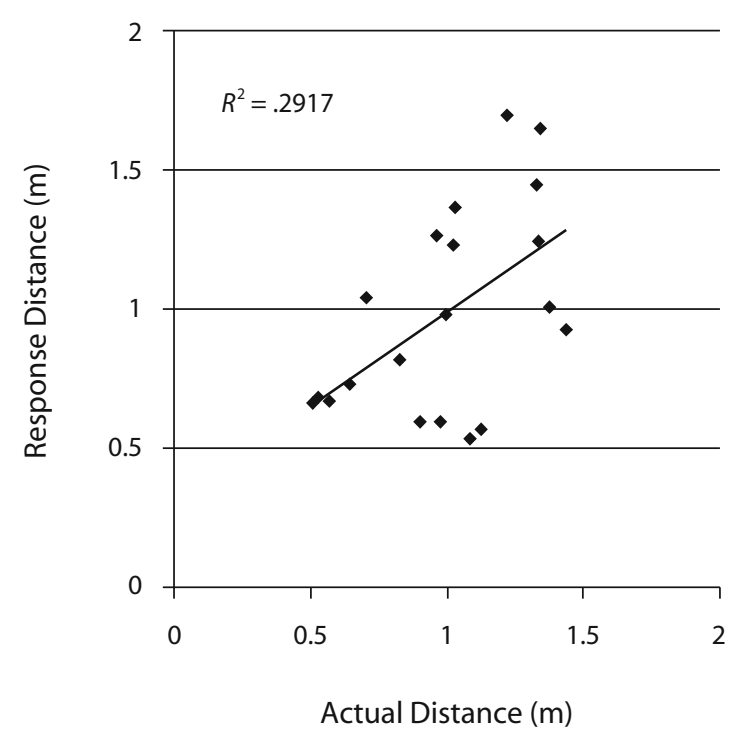

B.L.

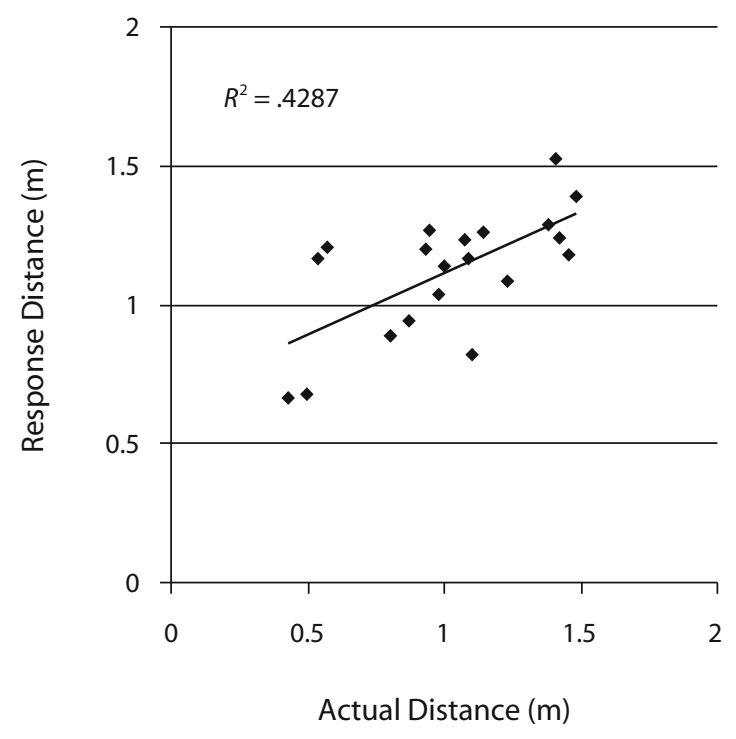

P.M.

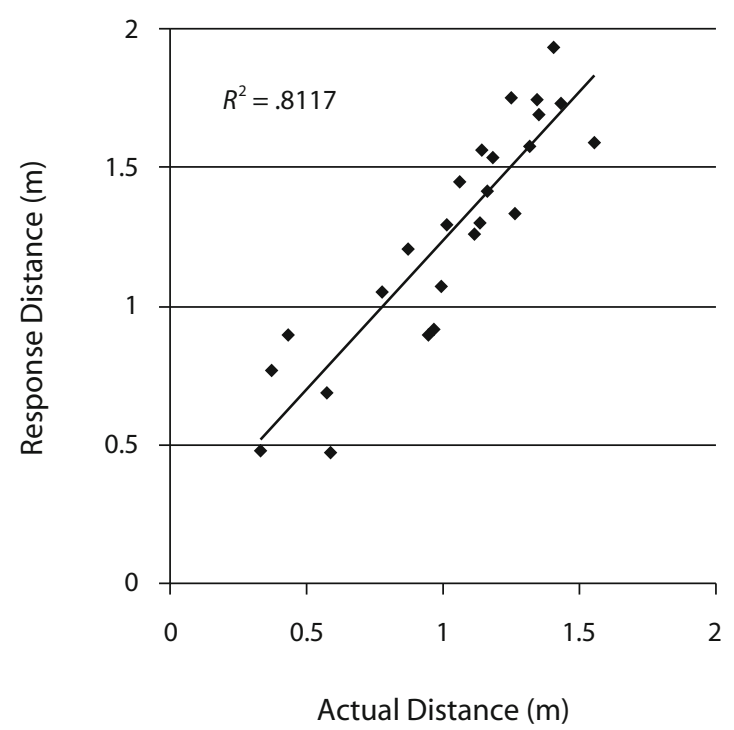

Figure 3. The distance response as a function of the actual distance for each participant. The solid lines are the regression lines.

$.63 ; t \mathrm{~s}>2.96 ; p \mathrm{~s}<.01)$. The 3 -D component showed similar effects (nonsignificant for E.C. $[\beta=0.27, t=$ $1.40, p=.18]$; significant for all others $[\beta \mathrm{s}=0.36,0.38$, $0.53 ; t \mathrm{~s}>2.14 ; p \mathrm{~s}<.05])$.

In the relative-orientation judgment task, all of the participants showed significant correlation between their responses and the actual angle $(r \mathrm{~s}=.83, .85, .54, .77 ; t \mathrm{~s}>$ 2.97; $p \mathrm{~s}<.01$; see Figure 4). A multiple linear regression on the responses as a function of the 3-D and 4-D components showed that the 4-D component was a significant predictor $(\beta \mathrm{s}=0.66,0.83,0.43,0.84 ; t \mathrm{~s}>2.17$; $p \mathrm{~s}<$ $.05)$, as well as the $3-\mathrm{D}$ component $(\beta \mathrm{s}=0.51,0.53,0.52$,
$0.62 ; t \mathrm{~s}>2.61 ; p \mathrm{~s}<.02)$. These data indicate that the participants incorporated both the 3-D and 4-D information into their judgments in both tasks.

\section{DISCUSSION}

These results suggest that humans are capable of representing and making basic spatial judgments on 4-D objects that they learn visually. There are several possibilities concerning the nature of these representations. The first possibility is that the representation is not spatial at all, but algebraic. That is, the participants remembered the 4-D coordinates 
F.W.

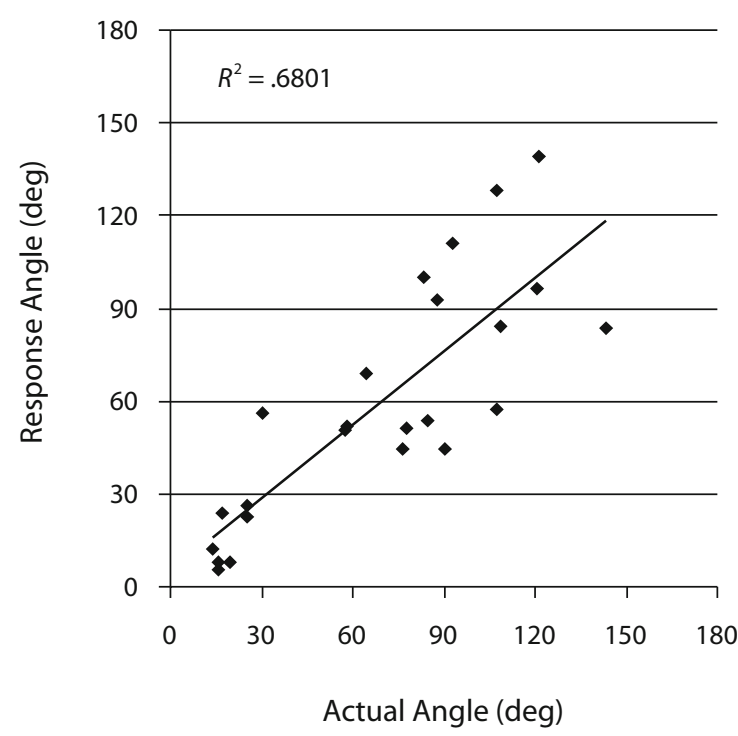

E.C.

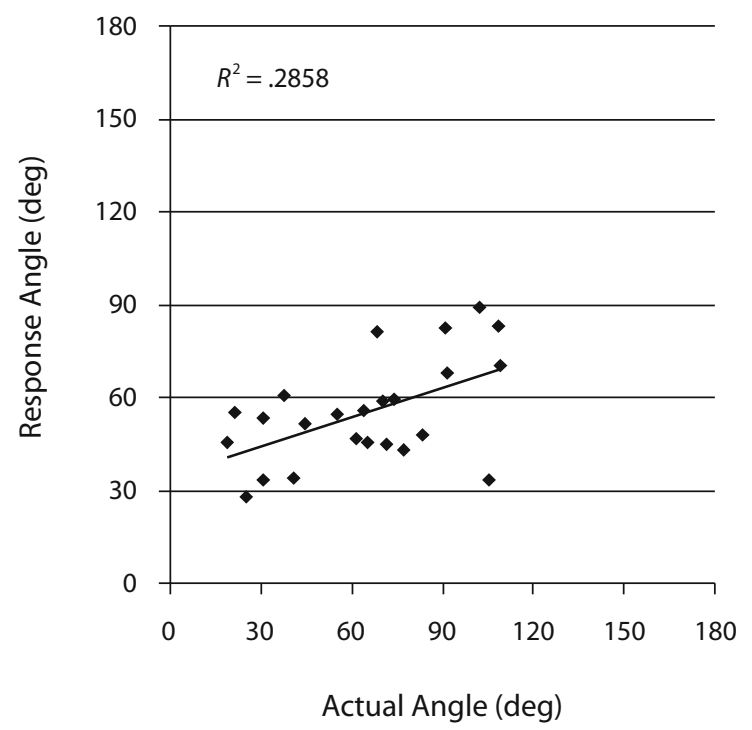

B.L.

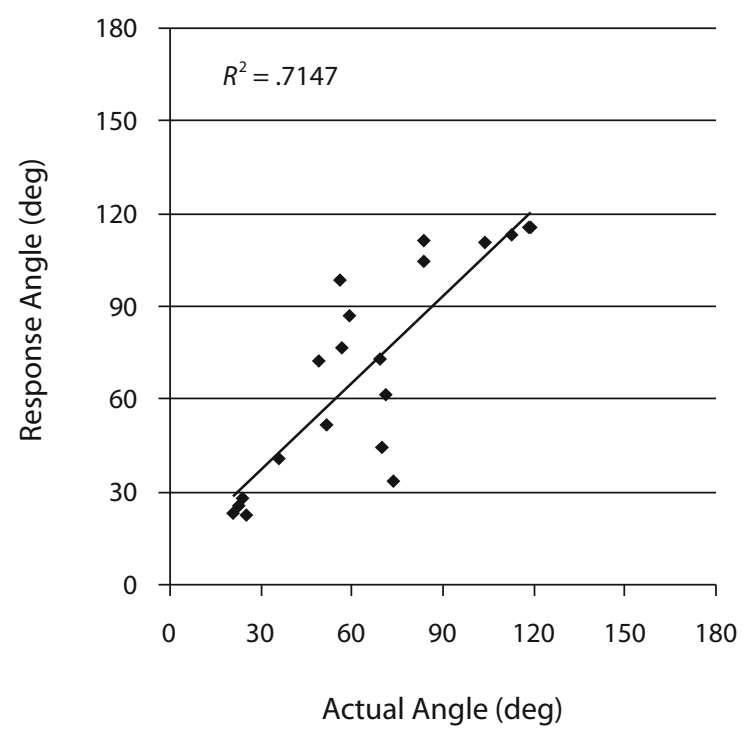

P.M.

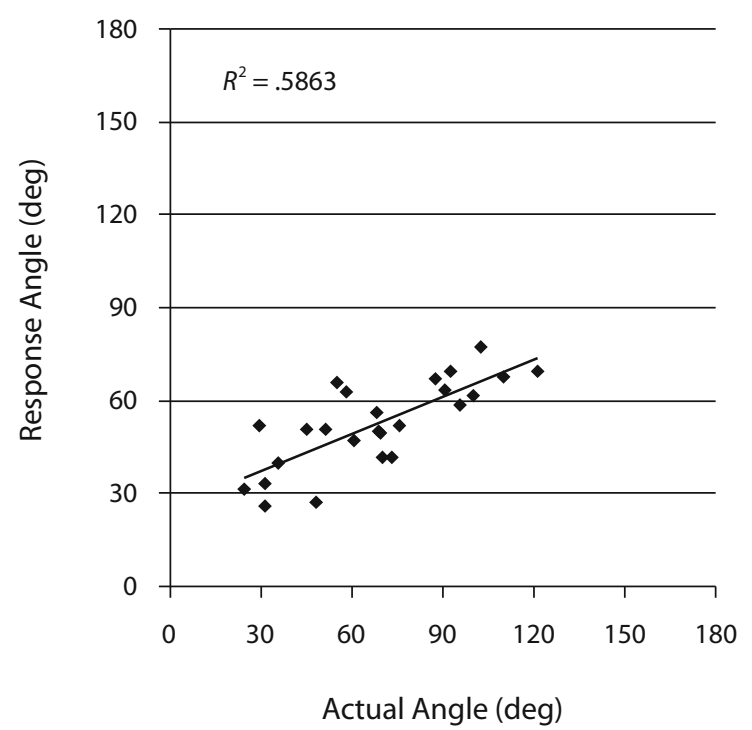

Figure 4. The relative-orientation response as a function of the actual angle for each participant. The solid lines are the regression lines.

of the marked vertices and then calculated the distance or angles algebraically. None of the participants reported using this strategy in either task. Furthermore, none of the participants even knew the exact formula for angle calculation, let alone were able to perform it by heart. Thus, it is unlikely that the representation is an algebraic one.

The second possibility is that the representation is spatial, but not 4-D. For example, the participants may have a 3-D-movie representation; that is, they simply remembered a sequence of 3-D sections of the 4-D object and made judgments based on these time-labeled 3-D representations. A 3-D movie is not a true 4-D spatial representation (because it is 3-D space + 1-D time). However, a 3-D-movie representation does not allow 4-D spatial judgments, because distance and angle are meaningless unless the time dimension is given a spatial interpretation and mapped onto a spatial dimension. Thus, the 3-D-movie representation may be (and very likely is) an intermediate stage, but the time dimension has to be converted to a spatial dimension eventually in order for the participants to perform the distance and angle judgment task.

The third possibility is that the representation is entirely spatial, but the fourth dimension is not really a new one but one that still lives in our 3-D world. For example, 
when the participants reported that they tried to form an image of the 4-D object by identifying the 3-D entrance point of each vertex and then imagining them being a certain hyperdepth away into some unknown space, they may be unknowingly imagining the vertices extending in the $z$ direction instead of a new, fourth one. This hypothesis is particularly plausible, given that the participants were physically walking along the $z$-axis while imagining themselves moving along the $w$-axis. Note that systematic biases would arise if the participants used the $z$-axis as the direction of hyperdepth, depending on the orientation of the target line relative to the $z$-axis. That is, the error in the 4-D distance estimation should be small when the 3-D projection of the target line was actually orthogonal to the $z$-axis, so that the $z$-axis became a legitimate substitute of the $w$ direction, and the error should be larger when the projection was more parallel to the $z$-axis. We examined the relationship between the distance-judgment error and the orientation of the 3-D projection of the target line relative to each of the three axes $(x, y$, and $z)$ for each participant, and we found no significant correlation between any of them $(r \mathrm{~s}<.33$, uncorrected $p \mathrm{~s}>.11$ for P.M.; $r \mathrm{~s}<.15$, uncorrected $p \mathrm{~s}>.52$ for the other participants). These results showed no evidence that the fourth direction that the participants were trying to imagine was one of the three existing axes, ${ }^{5}$ and thus they were consistent with the subjective reports that the participants solved the problem by imagining an object extended into a fourth dimension.

These data suggest that at least a subset of the population - both female and male - are capable of making 4-D distance and direction judgments. However, it is not clear how general this capability is. Other factors that can influence spatial judgment performances, such as expertise (e.g., in mathematics), gender, and individual differences (such as in spatial imagery abilities), may also influence one's acquisition of 4-D intuition.

The exact properties of these representations are also still unclear. The subjective reports indicate that mental construction of these 4-D objects was very effortful, and the results were difficult to sustain. The present data suggest that these representations can support judgments on the basic geometric properties of 4-D objects but are relatively weak and do not seem to achieve the level of vividness and clarity of ordinary mental imagery (Kosslyn, 1994). It is also unclear whether these representations can support more complicated spatial judgments and spatial processing, such as mental rotations. However, note that the participants in our study had minimal practice in these tasks, and it remains an open question whether it is possible to obtain more sustainable, definitive, and richer 4-D representations with increased perceptual experience in 4 -D virtual environments.

\section{AUTHOR NOTE}

This work was supported by NSF Grant BCS 03-17681 to R.F.W. The authors thank Blair Flicker for his programming assistance and Hank Kaczmarski and the Integrated Systems Laboratory at the Beckman Institute for technical support. Correspondence concerning this article should be addressed to R. F. Wang, Department of Psychology, University of Illinois. 603 E. Daniel St., Champaign, IL 61820 (e-mail: francesw@cyrus.psych.uiuc.edu).

\section{REFERENCES}

Аввотт, Е. А. (1991). Flatland: A romance of many dimensions. Princeton: Princeton University Press.

BERGER, D. (1965). Sphereland: A fantasy about curved spaces and an expanding universe. New York: Crowell.

Bork, A. M. (1964). The fourth dimension in nineteenth-century physics. Isis, 55, 326-338.

Davis, P. J., Hersh, R., \& Marchisotto, E. A. (1995). Four dimensional intuition. In The mathematical experience (pp. 442-447). Boston: Birkhäuser.

Durrell, F. (1938). Mathematical adventures. Boston: Humphries.

D'Zmura, M., Colantoni, P., \& Seyranian, G. (2000). Virtual environments with four or more spatial dimensions. Presence: Teleoperators \& Virtual Environments, 9, 616-631.

FinKe, R. A. (1993). Mental imagery and creative discovery. In B. Roskos-Ewoldsen, M. J. Intons-Peterson, \& R. E. Anderson (Eds.), Imagery, creativity, and discovery: A cognitive perspective (pp. 255285). Amsterdam: Elsevier Science.

FRANCIS, G. K. (2005). Metarealistic rendering of real-time interactive computer animations. In M. Emmer (Ed.), Mathematics and culture II (pp. 125-144). Berlin: Springer.

GARDNER, M. (1969). The unexpected hanging and other mathematical diversions. New York: Simon \& Schuster.

Gardner, M. (1975). Mathematical carnival. New York: Knopf.

KANT, I. (1896). Immanuel Kant's Critique of pure reason (F. M. Muller, Trans). London: Macmillans. (Original work published 1881)

KossLYN, S. M. (1994). Image and brain: The resolution of the imagery debate. Cambridge, MA: MIT Press.

ReICHENBACH, H. (1958). The philosophy of space and time. New York: Dover.

RUCKER, R. (1984). The fourth dimension: Toward a geometry of higher reality. Boston: Houghton Mifflin.

Seyranian, G. D. (2001). Human spatial perception in environments with four spatial dimensions. Dissertation Abstracts International, 62B, 1611.

SHePARD, R. N., \& COOPER, L. (1982). Mental images and their transformations. Cambridge, MA: MIT Press.

WeEKs, J. R. (1985). The shape of space. New York: Dekker.

\section{NOTES}

1. For information on the Beckman Institute Cube, see http://isl.uiuc .edu/Labs/CUBE/CUBE.html.

2. The 3-D slices were within a depth of $z=\{-0.6,0.6\}$. The observer moved between $z=-2.1$ and -0.9 .

3. Given two 4-D vertices $\mathrm{A}\left(x_{1}, y_{1}, z_{1}, w_{1}\right)$ and $\mathrm{B}\left(x_{2}, y_{2}, z_{2}, w_{2}\right)$, the $3-\mathrm{D}$ component of their distance was defined as the 3-D distance between $\left(x_{1}\right.$, $\left.y_{1}, z_{1}\right)$ and $\left(x_{2}, y_{2}, z_{2}\right)$, and the 4-D component was defined as $\left|w_{1}-w_{2}\right|$. Similarly, given three 4-D vertices $\mathrm{A}\left(x_{1}, y_{1}, z_{1}, w_{1}\right), \mathrm{B}\left(x_{2}, y_{2}, z_{2}, w_{2}\right)$, and $\mathrm{C}\left(x_{3}, y_{3}, z_{3}, w_{3}\right)$, the 3-D component of an angle in 4-D (e.g., between $\mathrm{AB}$ and $\mathrm{BC}$ ) was defined as the corresponding angle of their entrance points $\mathrm{A}^{\prime}\left(x_{1}, y_{1}, z_{1}\right), \mathrm{B}^{\prime}\left(x_{2}, y_{2}, z_{2}\right)$, and $\mathrm{C}^{\prime}\left(x_{3}, y_{3}, z_{3}\right)$ (e.g., between $\mathrm{A}^{\prime} \mathrm{B}^{\prime}$ and $\left.\mathrm{B}^{\prime} \mathrm{C}^{\prime}\right)$, and the 4-D component was defined as the difference between the actual angle and its 3-D component.

4. To strengthen the multiple regression analysis on the two components, the target objects were constructed to minimize the correlation between them. In the distance-judgment task, the 3-D components were randomly selected from the large range $(0.7-1.2 \mathrm{~m})$ in half of the trials and small range $(0.2-0.6 \mathrm{~m})$ in the other half. Within each set, the 4-D components were large $(0.7-1.2 \mathrm{~m})$ in half of the trials and small $(0.2-$ $0.6 \mathrm{~m}$ ) in the other half. Similarly, in the angle judgment task, the 3-D angular components were half large $\left(70^{\circ}-110^{\circ}\right)$ and half small $\left(20^{\circ}-50^{\circ}\right)$, and in each set, the 4-D components were half positive (between $10^{\circ}$ and $180^{\circ}$ ) and half negative (between $-180^{\circ}$ and $-10^{\circ}$ ). The resulting correlation between the two components was $r(85)=.10$ for the distance judgment task and $r(93)=-.26$ for the angle judgment task.

5. No effect was found when the data from all of the participants were combined. A further analysis using model fitting also failed to find evidence that the participants used an oblique line as the imagined direction.

(Manuscript received December 23, 2008; revision accepted for publication April 17, 2009.) 